\title{
A novel technique for performing gallbladder tumor biopsy using a stent delivery system and biopsy forceps
}

A 79-year-old man presented to our hospital with a gallbladder tumor diagnosed using abdominal ultrasonography (AUS). AUS, contrast-enhanced computed tomography, and magnetic resonance imaging showed a protruded lesion at the gallbladder fundus (> Fig.1, > Fig.2). Endoscopic retrograde cholangiography (ERC) was performed, and cholecystography revealed a contrast agent defect at the gallbladder fundus ( $>$ Fig. 3 ).

We placed a 0.025 -inch guidewire into the gallbladder and inserted a biliary stent delivery system, composed of a guide catheter and pusher tube (MAJ510; Olympus Medical Systems, Tokyo, Japan) (\$Fig.4). After removing the guidewire and guide catheter, we inserted biopsy forceps (radial Jaw 4 Pediatric Biopsy Forceps; Boston Scientific Japan, Tokyo, Japan) through the pusher tube and performed biopsy of the gallbladder fundus legion ( Video 1 ). The biopsy specimen was sufficient to diagnose gallbladder cancer ( $\mathbf{F i g . 5}$ ). Tumor recurrence was not seen for 3 years after surgery.

Although other endoscopic methods for diagnosing gallbladder cancers have been previously reported [1-3], use of biopsy forceps specimens for diagnosing gallbladder cancer is novel to our knowledge. We have previously reported the utility of a biliary stent delivery system in removing a foreign body from the bile duct $[4,5]$. The technique used here enabled us to insert biopsy forceps into the gallbladder safely and easily, and to obtain a sufficient biopsy sample. Moreover, this method may contribute to reducing complications after ERC, such as acute pancreatitis and duodenal perforation caused by the biopsy forceps injuring the duodenal papilla. Careful and accurate biopsy is important for prevention of gallbladder perforation. We placed an endoscopic naso-gallbladder drainage tube at the end of this procedure to reduce the

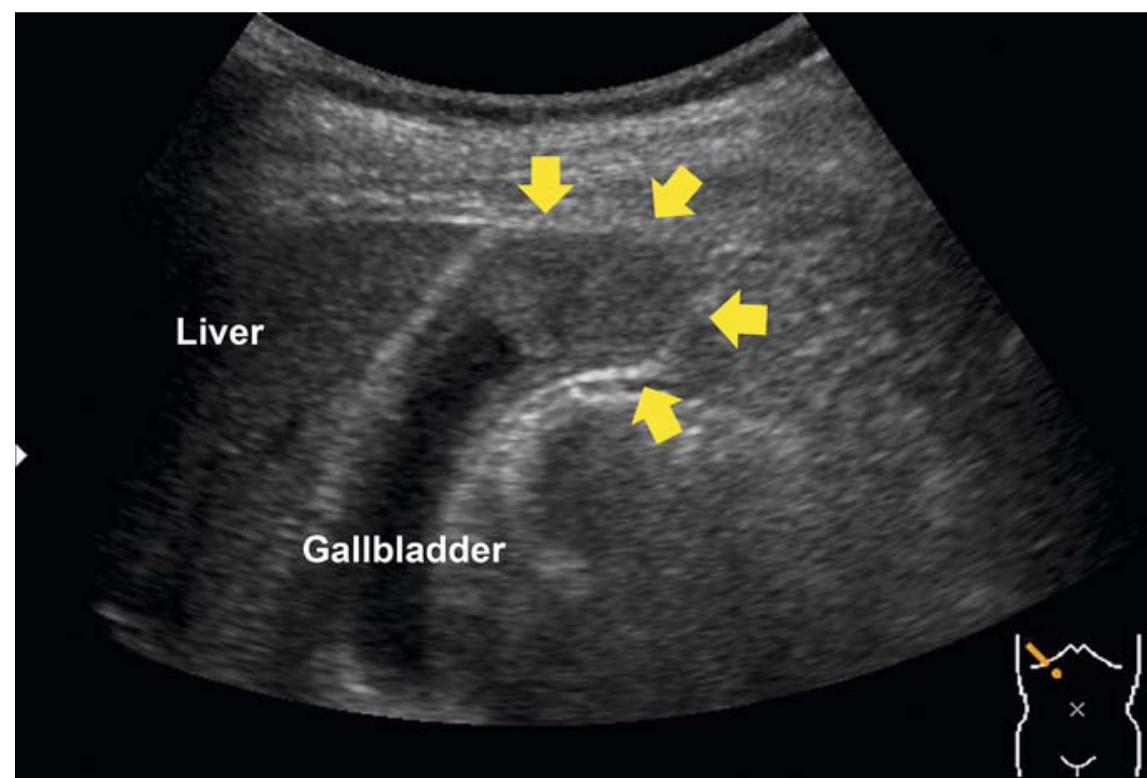

- Fig. 1 Abdominal ultrasonography of gallbladder cancer, showing a protruded lesion (yellow arrowheads) at the gallbladder fundus
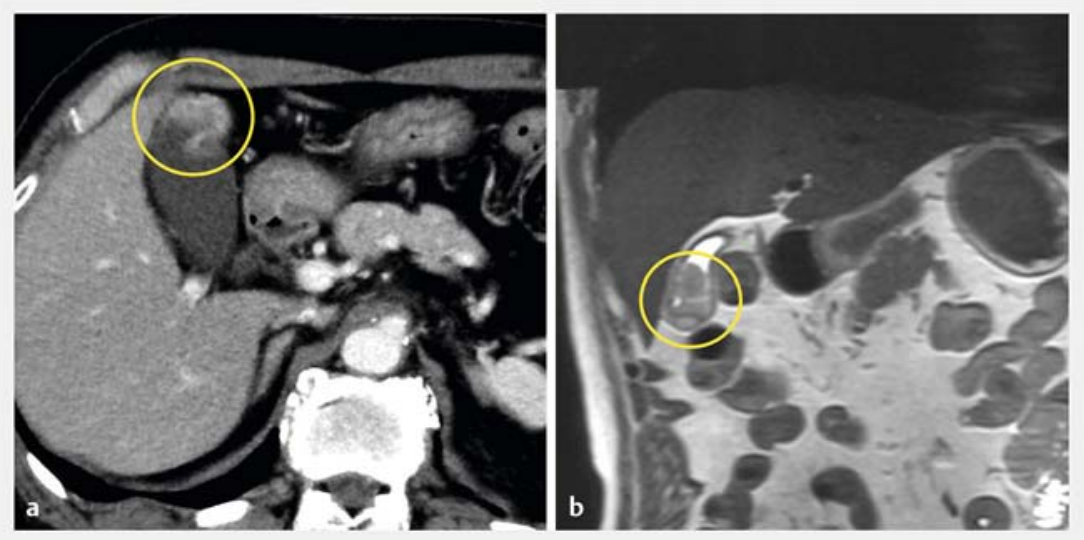

- Fig. 2 Imaging studies of gallbladder cancer showing a tumor lesion (yellow circle) at the gallbladder fundus. a Contrast-enhanced computed tomography. b Magnetic resonance imaging.

bile juice leakage in case of gallbladder Endoscopy_UCTN_Code_TTT_1AR_2AD perforation.

In conclusion, we report a novel technique to obtain a sample of the gallbladder using a stent delivery system using a biopsy forceps. 

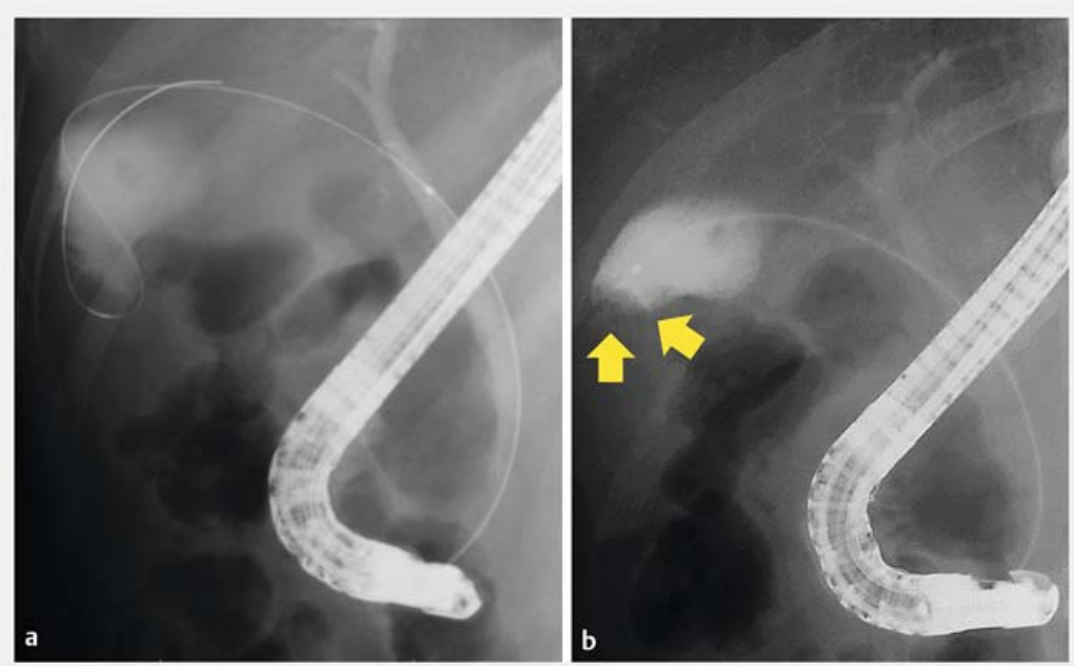

- Fig. 3 Endoscopic retrograde cholangiography. a A guidewire was inserted into the gallbladder. b Contrast agent defect (yellow arrows) was observed at the gallbladder fundus.
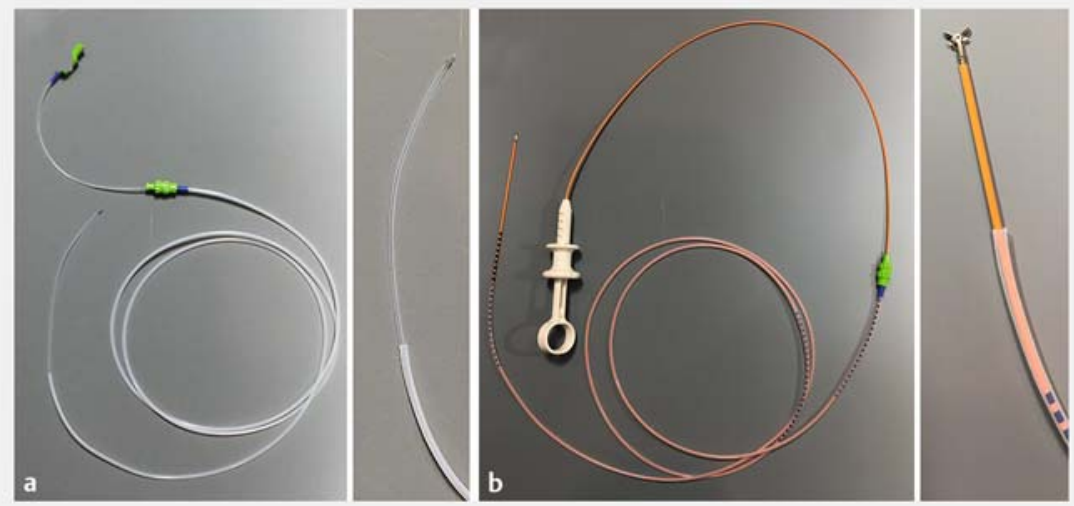

- Fig. 4 The biliary stent delivery system, composed of a guide catheter and pusher tube (MAJ-510; Olympus Medical Systems, Tokyo, Japan). a The biliary stent delivery system. b Biopsy forceps.
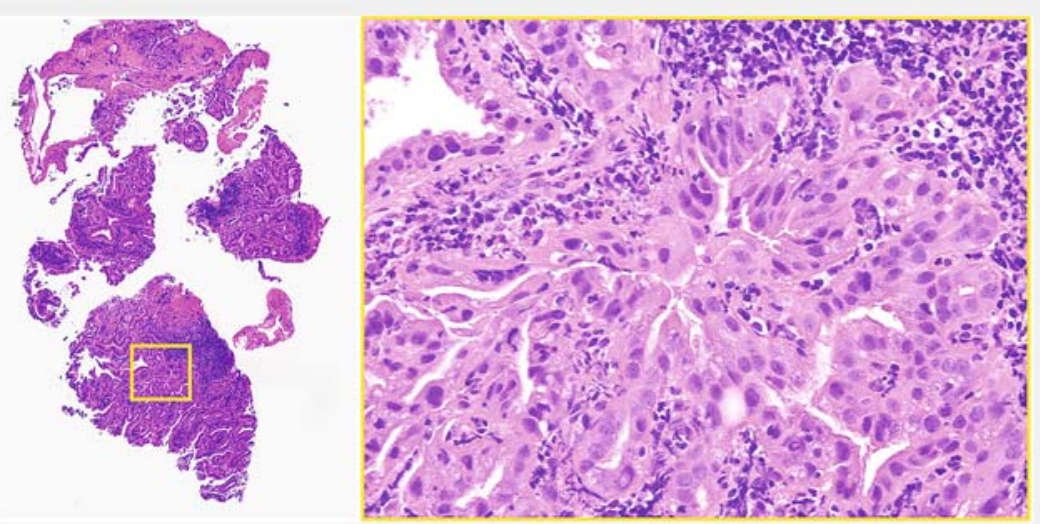

- Fig. 5 Hematoxylin and eosin staining of gallbladder cancer. The specimen was obtained from the gallbladder tumor using biopsy forceps. The histological diagnosis was adenocarcinoma.
The authors

Tomoaki Matsumori' ${ }^{1}$, Norimitsu Uza', Hirokazu Okada', Takahisa Maruno', Masahiro Shiokawa', Yuzo Kodama', ${ }^{1,}$, Hiroshi Seno $^{1}$

1 Department of Gastroenterology and Hepatology, Graduate School of Medicine, Kyoto University, Kyoto, Japan

2 Department of Gastroenterology, Graduate School of Medicine, Kobe University, Kobe, Japan

\section{Corresponding author}

\section{Norimitsu Uza, MD}

Department of Gastroenterology and Hepatology, Kyoto University Graduate School of Medicine, 54 Kawara-cho, Shogoin, Sakyo-ku, Kyoto, Japan

Fax: +81-75-7514302

uzanori@kuhp.kyoto-u.ac.jp

\section{References}

[1] Matsubayashi H, Kiyohara Y, Sasaki K et al. Metastatic malignant melanoma of the gallbladder diagnosed by cytology of endoscopic naso-gallbladder drainage fluid. J Dig Dis 2012; 13: 190-194

[2] Hammoud GM, Almashhrawi A, Ibdah JA et al. Usefulness of endoscopic ultrasoundguided fine needle aspiration in the diagnosis of hepatic, gallbladder and biliary tract lesions. World J Gastrointest Oncol 2014; 15: 420-429

[3] Hirata K, Kuwatani M, Suda G et al. A novel approach for the genetic analysis of biliary tract cancer specimens obtained through endoscopic ultrasound-guided fine needle aspiration using targeted amplicon sequencing. Clin Transl Gastroenterol 2019; 10: $\mathrm{e} 00022$

[4] Hijioka S, Hara K, Mizuno N et al. A novel technique for endoscopic transpapillary "mapping biopsy specimens" of superficial intraductal spread of bile duct carcinoma (with videos). Gastrointest Endosc 2014; 79 : 1020-1025

[5] Nishikawa Y, Uza N, Seno H et al. Successful endoscopic removal of fractured guidewire fragments from a peripheral bile duct using a biliary stent delivery system and biopsy forceps. Endoscopy 2018; 50: E279-E280 


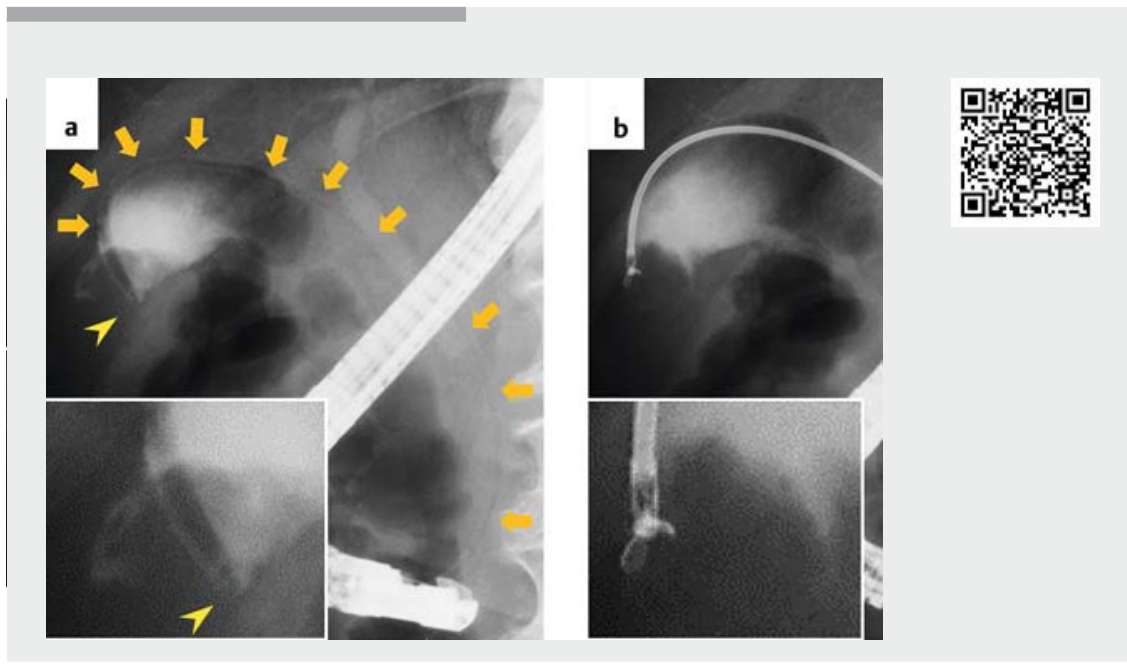

$\checkmark$ Video 1 Biopsy of gallbladder tumor. a A pusher tube was inserted into the gallbladder (orange arrows) and the tip of the tube (yellow arrowhead) was located at the gallbladder tumor. b Biopsy forceps was inserted into the gallbladder through the pusher tube.

\section{Bibliography}

Endoscopy 2020; 52: E415-E417

DOI $10.1055 / \mathrm{a}-1149-8625$

ISSN 0013-726X

published online 24.4.2020

(c) 2020. Thieme. All rights reserved.

Georg Thieme Verlag KG, Rüdigerstraße 14, 70469 Stuttgart, Germany

\section{ENDOSCOPY E-VIDEOS}

https://eref.thieme.de/e-videos

回回 Endoscopy E-Videos is a free

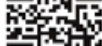
access online section, reporting 田: on interesting cases and new techniques in gastroenterological endoscopy. All papers include a high quality video and all contributions are freely accessible online.

This section has its own submission website at

https://mc.manuscriptcentral.com/e-videos 